\title{
Calculating Optimal Trajectories from Contact Transitions
}

\author{
Jun Takamatsu Hiroshi Kimura $†$ Katsushi Ikeuchi \\ Institute of Industrial Science \\ †Graduate School of Information Systems \\ The University of Tokyo \\ University of Electro-Communications \\ Tokyo, 106-8558, Japan \\ Tokyo, 182-8585, Japan
}

\begin{abstract}
The learning-from-demonstration method is focused on for a novel robot-programming style. It consists of two parts: to recognize human performance from observation as sequential motion primitives and to execute the same performance. We already proposed a method to recognize assembly tasks. However, execution requires the ability to convert motion primitives to collision free paths. In this paper, we describe a method to calculate collision free paths.

Many researchers have proposed to calculate collision free paths using analytical methods, potential fields or probabilistic methods. Potential and probabilistic methods are very powerful tools on a computer, but their solutions are not optimal. We propose a method to calculate optimal collision free paths analytically.
\end{abstract}

\section{Introduction}

Recently, the method called "Learning from demonstration," an easy robot-programming style, has been focused on $[1,2,3,4,5,6,7]$. Its main characteristic is that it is enough to perform a desired action in front of the robot's vision system to make it repeat the action. Therefore, even people with little robotics knowledge can use the method. It recognizes an action as a sequence of symbolic motion primitives. Symbolization enables a robot to execute the same action more flexibly. We proposed a method to symbolize and sequence assembly tasks[8].

The "Learning from Demonstration" method requires control parameters for a robot to perform the desired actions, because the actions have already been converted to symbolic motion primitives, that do not have such parameters. In this paper, we especially consider trajectories.

First, trajectories must be collision-free. LozanoPerez et al. proposed a method to generally calculate collision-free trajectories using the concept of Configuration Space(C-space)[9]. However, it may be impossible to analytically calculate such trajectories in a high-dimensional C-space, even for six dimensions, which is equivalent to our living world. Kavraki et al. proposed the "Probabilistic Roadmap Method(PRM)[10]" to calculate such trajectories using a randomized algorithm.

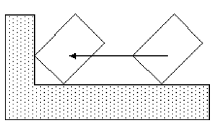

(a)

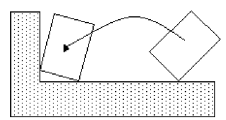

(b)
Figure 1: Optimal trajectory

Next, increasing the success rate of assembly tasks usually requires calculating a trajectory that maintains a contact relation. Hirukawa proposed a method to analytically formulate and calculate a trajectory in a spatial motion case[11]. However, it is not always possible to solve such formulations, the complexity of the problem depending on the user's skill at choosing adequate ones. Ji and Xiao proposed a method to solve the formulations using PRM[12].

We propose a method to analytically and easily calculate an optimal trajectory by first calculating an axis direction of rotation. The method is based on the fact that we prefer the rotation in which the direction of the axis is constant.

The reader may wonder why we solve a trajectory by such a method, instead of simply using PRM.

Consider the case as shown in Figure 1. The two trajectories, (a) and (b), satisfy the condition to move to the next contact transition maintaining the contact relation. However, the trajectory (b) includes wasteful motion. Although the PRM method may not be able to find the waste, our method can, because the trajectory obtained by our method is equal to the trajectory (a).

The contents of this paper are as follows: Section 2 easily illustrates our method to recognize assembly tasks proposed in [8]. Section 3 presents the method to calculate optimal trajectories. Section 4 verifies the validity of the method. Section 5 concludes this paper.

\section{Recognition of assembly tasks}

In [8], we proposed a method to describe assembly tasks as a sequence of "sub-skills". These are essential motion primitives in assembly tasks, representing transitions between contact relations.

Performing assembly tasks means to achieve the de- 


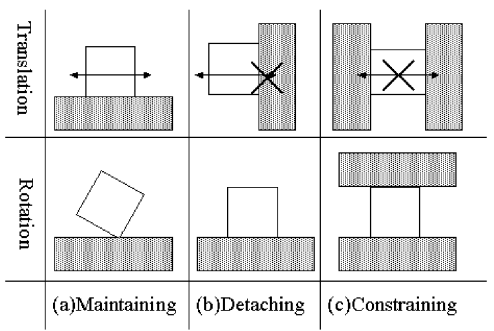

Figure 2: Maintaining, detaching, and constraining degree of freedoms(DOFs)

sired transitions. Because infinite contact relations and transitions exist, it is impossible to recognize assembly tasks deductively. Therefore, it is necessary to analyze contact relations and transitions.

First, we focus on the relationship between a contact relation and feasible motion of a grasped object, and we analyze a contact relation using the concept of a motion degree of freedom(DOF). Next, we consider the design of sub-skills from often occurring transitions. Then, we describe the method to recognize assembly tasks.

Some assumptions exist as follows; 1) All objects are polyhedral and rigid. 2) Only two objects exist, one is a grasped object and the other is referred to as an environmental object. 3) The environmental object is fixed.

\subsection{Motion DOF}

In translation and rotation, three kinds of motion DOFs can be defined as follows (shown in Figure $2)[8]$;

\section{Translation}

Maintaining the DOF along which an object can translate maintaining a contact relation.

Detaching the DOF along which an object can translate not maintaining a contact relation.

Constraining the DOF along which an object cannot translate.

\section{Rotation}

Maintaining the DOF of an axis around which an object can rotate maintaining a contact relation.

Detaching the DOF of an axis around which an object can rotate not maintaining a contact relation.

Constraining the DOF of an axis around which an object cannot rotate.

Given a contact relation, the six DOFs of an object configuration can be classified into three types of motion DOFs using the feasible motion in C-space[9]. However, motion must be formulated by simultaneous non-linear inequalities and it is very difficult to classify six DOFs using these inequalities. Therefore, we try to classify the six DOFs using infinitesimal feasible motion, that is, simultaneous inequali-

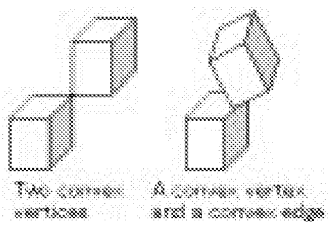

Figure 3: Two types of singular contact-elements.

ties linearized by the screw theory $[13]^{1}$.

It is known that a contact relation between two polyhedral objects can be represented as a combination of vertex-face $(\mathrm{V}-\mathrm{F})$, face-vertex $(\mathrm{F}-\mathrm{V})$, and non-parallel edge-edge (E-E) contacts ${ }^{2}$. We can formulate the infinitesimal feasible motion as equation (1), where $\mathbf{A}_{\mathbf{i}}$ is a six dimensional vector and $\mathbf{T}=\left(t_{1}, \ldots, t_{6}\right)$.

$$
\cap_{i} \mathbf{A}_{\mathbf{i}} \cdot \mathbf{T} \geq 0
$$

\subsection{Analysis of a contact relation}

We classify six DOFs into motion DOFs as follows:

1. The formulation relating only to translation is extracted from equation (1).

2. The formulation relating to the direction of a rotation axis is extracted from equation (1).

[8] describes more details.

\subsection{Singular contact relation}

Consider the case that the shape of infinitesimal feasible motion in $\mathrm{C}$-space is concave. The motion cannot be represented as equation (1), because the shape is not convex. However, it can be represented as equation (2), where $\mathbf{B}_{\mathbf{i j}}$ is a six dimensional vector.

$$
\left(\cap_{i} \mathbf{A}_{\mathbf{i}} \cdot \mathbf{T} \geq 0\right) \cap\left(\cap_{i} \cup_{j} \mathbf{B}_{\mathbf{i j}} \cdot \mathbf{T} \geq 0\right)
$$

Such a case happens if and only if a contact as shown in Figure 3, referred to as a singular contact, is included in a contact relation.

In this case, we analyze the contact relation obtained by removing singular contacts to an original contact relation. Especially, we refer to these motion DOFs as singular maintaining, singular detaching, and singular constraining DOFs.

$$
\cap_{i} \mathbf{A}_{\mathbf{i}} \cdot \mathbf{T} \geq 0
$$

Unfortunately, this simplification of the analysis sometimes causes different types of motion DOFs to be classified into the same type. [8] shows how to correct the analysis. As it is not of importance for this paper, we do not describe it in more details.

\footnotetext{
${ }^{1}$ The screw theory is the first order Taylor expansion of feasible motion. Rimon and Burdick proposed to use higher order terms[14]. But they introduced them in form of an index of immobility, not of higher order motion DOFs, which makes it hard to apply their method directly to our system. We think that the screw theory is sufficient for our goals.

${ }^{2} \mathrm{~A}$ V-F contact means that a vertex of the grasped object is contact with a face of the environmental object.
} 


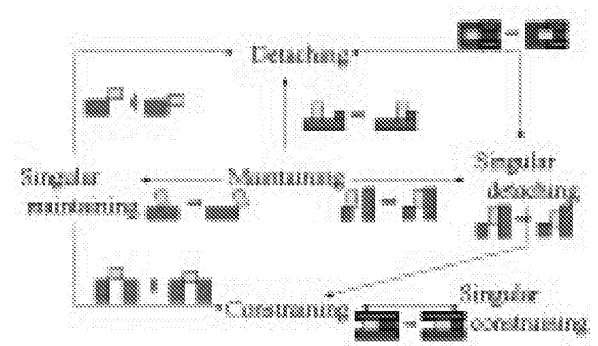

Figure 4: Possible transitions

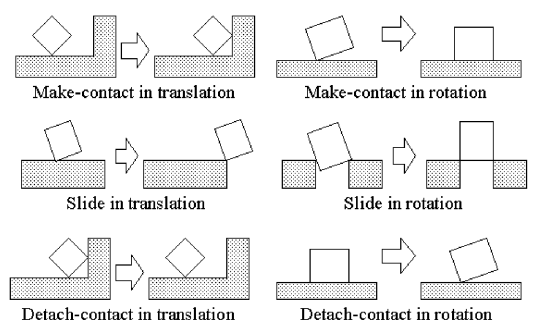

Figure 5: Class 1 sub-skills

\subsection{Transitions of motion DOFs and sub- skills}

A transition between two contact relations leads to one of the motion DOFs. Six types of motion DOFs exist in translation and rotation. If we assume that the motion to maintain a singular contact-relation does not exist ${ }^{3}$, only 16 types of transitions usually occur as shown in Figure 4[8].

These 16 transitions can be classified into two types: One includes six transitions between maintaining and detaching, between maintaining and singular maintaining, and between maintaining and singular detaching. These transitions have the characteristic that the direction of the motion is the same as the direction of the motion DOF. We refer to the motion leading to these transitions as a class 1 subskill. Considering these transitions, we can define six types of class 1 sub-skills as shown in Figure 5.

The other includes all remaining transitions. These transitions have the characteristic that the direction of the motion is different from the direction of the motion DOF. We refer to the motion leading to these transitions as a class 2 sub-skill. These sub-skills represent a condition to be satisfied to translate to the next contact relation. In detail, they are classified into three types as follows; The first type needs to precisely control an object configuration to translate to the next contact relation (Shown in Figure 6(a)). The second type needs not to precisely control an object configuration (Shown in Figure 6(b)(c)). The third type is lead by a slide sub-skill (Shown in Figure $6(d))$.

\footnotetext{
${ }^{3}$ The purpose of this paper is to recognize human assembly tasks. Because we usually avoid this motion in assembly tasks, the assumption is valid.
}

(a)
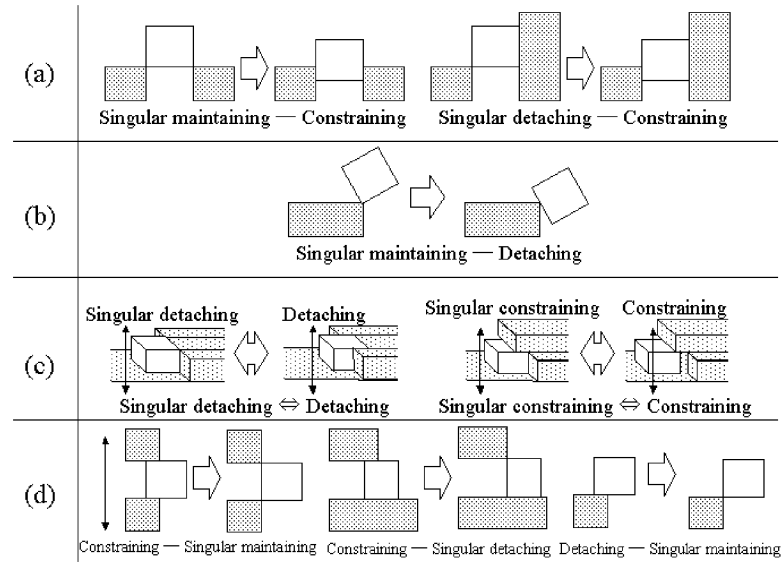

Figure 6: Class 2 sub-skills

\section{Generation of optimal trajectories}

Class 2 sub-skills mainly represent a condition for a transition to the next contact relation, therefore, we need only to consider class 1 sub-skills to generate optimal trajectories.

Usually detach-contact sub-skills seldom appear because the process of assembly usually makes the constraint of the motion ${ }^{4}$ increase. Trajectories of detach-contact sub-skills can be calculated as inverse trajectories of make-contact sub-skills.

Make-contact and slide sub-skills only differ from each other in the condition to decide when sub-skills finish. Therefore, in both sub-skills, the optimal trajectories can be calculated by the method mentioned below.

First, we consider the case that the constraint increases in translation only. Next, we consider the case that the constraint increases in rotation only. After that, we consider the case that the constraint increases in both translation and rotation.

\subsection{Translation}

In the case that the constraint increases in only translation, the desired transition can be achieved by translation only. For simplification, we consider the environmental object coordinate frame as the standard one. Let $\mathbf{q}$ be the configuration of the grasped object, and let $C_{b}=\left\{c b_{1}, \ldots, c b_{l}\right\}$ and $C_{e}=\left\{c e_{1}, \ldots, c e_{m}\right\}$ be the contact relations at the beginning and the end of the transition, where $c b_{i}, c e_{i}$ are $\mathrm{V}-\mathrm{F}, \mathrm{F}-\mathrm{V}$, or E-E contacts.

The displacement of a grasped object $\Delta \mathbf{T}$ must maintain the contact relation $C_{b}$. That condition is represented by equation $(4)^{5}$, where $\mathbf{n}_{\mathbf{i}}$ is a face normal of $F_{i}$ if $c b_{i}$ is a $V_{i}-F_{i}$ or $F_{i}-V_{i}$ contact, and a vector which is perpendicular to each edge if $c b_{i}$ is

\footnotetext{
${ }^{4}$ The constraint of a motion can be regarded as the sum of detaching and constraining DOFs.

${ }^{5}$ Equation (4) is constant if the object orientation is constant.
} 


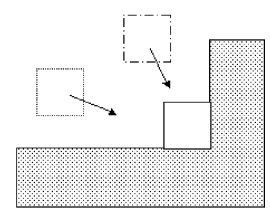

Figure 7: Redundant solution in translation

an E-E contact, when the orientation of the grasped object is $\mathbf{q}$.

$$
\cap_{i=1}^{l} \mathbf{n}_{\mathbf{i}} \cdot \Delta \mathbf{T}=0
$$

When the dimension of the solution $\Delta \mathbf{T}$ is zero, that is, the solution $\Delta \mathbf{T}$ is only $\mathbf{0}$, the grasped object cannot translate.

Next, when the dimension is one, $\Delta \mathbf{T}=s\left(\mathbf{n}_{\mathbf{p}_{\mathbf{1}}} \times\right.$ $\mathbf{n}_{\mathbf{p}_{\mathbf{2}}}$ ), where $\mathbf{n}_{\mathbf{p}_{\mathbf{1}}}, \mathbf{n}_{\mathbf{p}_{\mathbf{2}}}$ are two linear independent vectors of $\left\{\mathbf{n}_{\mathbf{1}}, \ldots, \mathbf{n}_{\mathbf{l}}\right\}$ and $s$ is any real number satisfying the condition $\Delta \mathbf{T} \cdot \Delta \mathbf{T}_{\mathbf{o}} \geq 0$, and $\Delta \mathbf{T}_{\mathbf{o}}$ is the observed displacement.

When the dimension is two or three, various solutions exist. We calculate optimal trajectories by employing a characteristic of a class 1 sub-skill that the direction of the motion is closely related to the direction of the transition.

Let $\Delta \mathbf{T}_{\mathbf{b}}$ be the feasible displacement in the beginning contact relation (that is, the solution of equation (4)) and $\Delta \mathbf{T}_{\mathbf{e}}$ be the feasible displacement in the end contact relation (that is, the solution of equation (4), when the orientation of the grasped object is $\mathbf{q}$ and the contact relation is $C_{e}$ ). The space $\Delta \mathbf{T}_{e}$ must be a linear sub-space of $\Delta \mathbf{T}_{\mathbf{b}}$. Considering the characteristics of a class 1 sub-skill, the optimal displacement $\Delta \mathbf{T}$ can be obtained by subtracting $\mathbf{T}_{\mathbf{e}}$ from $\mathbf{T}_{\mathbf{s}}$, as expressed in equation (5), where $\dot{+}$ represents the direct sum.

$$
\Delta \mathbf{T}_{s}=\Delta \mathbf{T}_{e} \dot{+} \Delta \mathbf{T}
$$

When the dimension of $\Delta \mathbf{T}$ is one, it is the optimal displacement. Next, when the dimension is two or three, the optimal displacement cannot be decided by equation (5), because it depends on the location of the grasped object as shown in Figure 7.

In such a case, the optimal displacement may be equal to the straight line between object configurations at the beginning and at the end of the transition without changing the orientations. Object configurations at the beginning and end obtained from observation usually have different orientations. By applying the method for vision error correction, as proposed in [15], we can calculate object configurations with the same orientations. The problem is whether the displacement between such object configurations is optimal or not.

When the dimension of $\Delta \mathbf{T}$ is two or three, the dimensions of $\Delta \mathbf{T}_{\mathbf{b}}$ and $\Delta \mathbf{T}_{\mathbf{e}}$ must be $(3,0),(3,1)$, or $(2,0)$. In the case they are $(3,0)$ and $(2,0)$, the object configuration at the end is uniquely decided when an object orientation is decided. Therefore, the displacement between the two object configurations is optimal. In the case $(3,1)$, the displacement may not be optimal, however, we can easily calculate the optimal displacement by calculating the optimal object configuration $\mathbf{L}_{\mathrm{e}}^{\prime}$ at the end by equation (6), where $\mathbf{L}_{\mathbf{b}}$ and $\mathbf{L}_{\mathbf{e}}$ are the object configurations at the beginning and end with the same orientation.

$$
\mathbf{L}_{e}^{\prime}=\mathbf{L}_{e}-\frac{\Delta \mathbf{T}_{\mathbf{e}} \cdot\left(\mathbf{L}_{\mathbf{e}}-\mathbf{L}_{\mathbf{b}}\right)}{\left|\Delta \mathbf{T}_{\mathbf{e}}\right|^{2}}
$$

The optimal displacement is $s\left(\mathbf{L}_{\mathbf{e}}^{\prime}-\mathbf{L}_{b}\right)$.

\subsection{Rotation}

Aside from translation, the motion comprises only rotation. However, the rotation is defined as one in which the axis direction is constant ${ }^{6}$.

First, we calculate the direction of the rotation axis from the result of assembly task recognition. Next, we describe an equation to represent the relationship between the object configuration and the amount of rotation.

Direction of the rotation axis. From the process of the recognition, we can obtain the equation for the direction of the rotation axis as shown in equation (7), where $\mathbf{r}_{\mathbf{b}}$ and $\mathbf{r}_{\mathbf{e}}$ are the axis directions at the beginning and end of the contact relations.

$$
\cap_{i} \mathbf{a s}_{\mathbf{i}} \cdot \mathbf{r}_{\mathbf{b}}=0, \cap_{i} \mathbf{a e}_{\mathbf{i}} \cdot \mathbf{r}_{\mathbf{e}}=0
$$

The solution of $\mathbf{r}_{\mathbf{b}}$ in equation (7) includes the desired axis direction. Furthermore, because the solution of $\mathbf{r}_{\mathbf{e}}$ is a linear sub-space of $\mathbf{r}_{\mathbf{b}}$, it is possible to calculate the axis direction by the same method as the translation.

However, the axis direction obtained by straight interpolation between the beginning and the end may not be optimal, because the formulations of $\mathbf{r}_{\mathbf{b}}$ and $\mathbf{r}_{\mathbf{e}}$ are dependent on an object configuration obtained from observation.

First, when the dimension of solution $\mathbf{r}_{\mathbf{b}}$ is zero, the grasped object cannot rotate. Next, when the dimension is one, the direction of the rotation axis is optimal.

The problem is when the dimension of $\mathbf{r}_{\mathbf{b}}$ is two or three. First, when the dimension of $\mathbf{r}_{\mathbf{e}}$ is zero, the optimal axis direction is a simple straight interpolation. Naturally, the optimal direction should be one of the solutions of $\mathbf{r}_{\mathbf{b}}$.

In the other cases, we need to decide on the essential rotation. We describe the decision method later.

Amount of rotation and object configuration. We consider the environmental object coordinate

\footnotetext{
${ }^{6}$ In this paper, motion is classified into three types as follows: translation, rotation and another motion. However, the third one seldom appears, therefore, we ignore it here.
} 

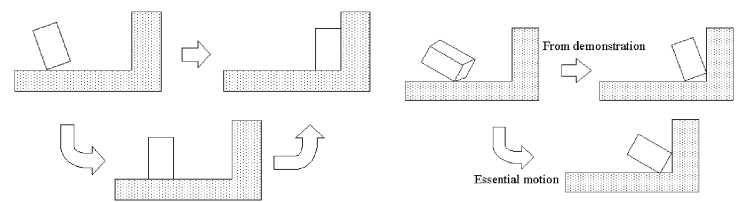

Figure 8: Translate and rotate simultaneously

Figure 9: Essential motion

frame as the standard one. A grasped object configuration q must satisfy the simultaneous non-linear equalities (8) to maintain a present contact relation $C_{b}{ }^{7}$, where $\mathbf{v}_{g_{i}}, \mathbf{n}_{g_{i}}, \mathbf{f}_{g_{i}}$ are the vertex position, the face normal, and any point on the face of the grasped object in the grasped object coordinate frame, $\mathbf{e}_{m_{i}}, \mathbf{p}_{m_{i}}$ are the vertex position, the face normal, and any point on the face of the environmental object in the environmental object coordinate frame, $\mathbf{e}_{m_{i}}$ and $\mathbf{p}_{m_{i}}$ are the edge direction and any point on the edge of a grasped object in the grasped object coordinate frame, $\mathbf{e}_{e_{i}}$ and $\mathbf{p}_{e_{i}}$ are the edge direction and any point on the edge of the environmental object in the environmental object coordinate frame, $R_{\text {org }}$ is a $3 \times 3$ matrix representing the initial orientation of the grasped object, $\mathbf{T}$ is a $3 \times 1$ matrix representing the location of the grasped object, and $I$ is a $3 \times 3$ unit matrix.

$$
\begin{gathered}
\cap_{i=1}^{l} f_{i}(\mathbf{q})=0 \\
f_{i}(\mathbf{q})=\left\{\begin{array}{r}
\mathbf{n}_{\mathbf{e}_{\mathbf{i}}} \cdot\left(R \mathbf{v}_{\mathbf{g}_{\mathbf{i}}}+\mathbf{T}-\mathbf{f}_{\mathbf{e}_{\mathbf{i}}}\right) \\
\left(\text { when } c b_{i} \text { is a V-F contact }\right) \\
R \mathbf{n}_{\mathbf{g}_{\mathbf{i}}} \cdot\left(\mathbf{v}_{\mathbf{e}_{\mathbf{i}}}-R \mathbf{f}_{\mathbf{g}_{\mathbf{i}}}-\mathbf{T}\right) \\
\left(\text { when } c b_{i} \text { is an F-V contact }\right) \\
\left(R \mathbf{e}_{\mathbf{g}_{\mathbf{i}}} \times \mathbf{e}_{\mathbf{e}_{\mathbf{i}}}\right) \cdot\left(\mathbf{p}_{\mathbf{e}_{\mathbf{i}}}-R \mathbf{p}_{\mathbf{g}_{\mathbf{i}}}-\mathbf{T}\right) \\
\left(\text { when } c b_{i} \text { is an E-E contact }\right)
\end{array}\right. \\
R=R_{a x i s} R_{\text {org }} \\
R_{\text {axis }}=E+[\mathbf{r}]_{\times} \sin \Delta \theta+[\mathbf{r}]_{\times}^{2}(1-\cos \Delta \theta) \\
{[\mathbf{r}]_{\times}=\left(\begin{array}{ccc}
0 & -r_{z} & r_{y} \\
r_{z} & 0 & -r_{x} \\
-r_{y} & r_{x} & 0
\end{array}\right)}
\end{gathered}
$$

Robot control is not continuous but discrete because of computer limitations, so we need only to quickly calculate the grasped object configuration given an amount of rotation angle. We already calculated the direction of the rotation axis, therefore, the calculation of the object configuration is equivalent to solving redundant simultaneous linear equalities. These can be easily solved.

Both translation and rotation. In the case that the constraint in both translation and rotation increases, an object must both translate and rotate to achieve the next contact relation. However, as shown in Figure 8, at first a grasped object rotates to achieve the desired orientation, then it translates to

\footnotetext{
${ }^{7}$ Moreover, an equation representing not only that the length is zero between contact elements but also a possible range must be satisfied. However, we ignore this here.
}

achieve the next contact transition ${ }^{8}$. The desired rotation and translation can be calculated by the above mentioned method.

\subsection{Essential rotation}

In the contact transition as shown in Figure 9, the essential rotation must be one as shown in the lower right of the figure. We describe a method to calculate the essential rotation, given object configurations at the beginning and the end, obtained from observation.

Let the $3 \times 3$ matrices, $R_{b}$ and $R_{e}$, represent the grasped object orientation at the beginning and the end in the environmental object coordinate frame. Then, we can calculate the essential rotation using the following algorithm:

1. consider the coordinate frame (referred to as an axis coordinate frame) in which the $\mathrm{x}-, \mathrm{y}-$, and $\mathrm{z}$-axes correspond to the axes around which the grasped object can rotate.

2. convert the object configurations in the environmental object coordinate frame to ones in the axis coordinate frame.

3. obtain the displacement matrix from the beginning to the end in the axis coordinate frame.

4. convert the displacement matrix to RPYrepresentation.

5. obtain the essential displacement matrix that sets the amount of unessential rotation axes zero.

6. calculate the axis direction and amount of rotation by the essential displacement.

Let $n_{b}$ and $n_{e}$ be the numbers of axis DOFs, that is, the dimensions of solution $\mathbf{r}_{\mathbf{b}}$ and $\mathbf{r}_{\mathbf{e}}$ in equation (7). The condition, $0<n_{e}<n_{s} \leq 3$, must be satisfied $^{9}$. The solution of $\mathbf{r}_{\mathbf{b}}$ can be represented by a linear sum of the vectors, $\mathbf{r}_{\mathbf{1}}, \ldots \mathbf{r}_{\mathbf{n}_{\mathrm{b}}}$, and the solution of $\mathbf{r}_{\mathbf{e}}$ represented by a linear sum of the vectors, $\mathbf{r}_{1}, \ldots, \mathbf{r}_{\mathbf{n}_{\mathrm{e}}}$, because the solution space of $\mathbf{r}_{\mathrm{e}}$ must be a linear sub-space of $\mathbf{r}_{\mathbf{s}}$.

The essential rotation can be obtained by removing the rotation around the axes, $\mathbf{r}_{\mathbf{1}}, \ldots, \mathbf{r}_{\mathbf{n}_{\mathbf{e}}}$ from the rotation obtained form observation. Let a $3 \times 3$ matrix $R_{a}$ convert the environmental object coordinate frame to the axis coordinate frame, where the $x_{-}^{-}, y_{-}^{-}$, and $\mathrm{z}$-axes correspond to axes as shown in Table 1 . Then, the object configurations at the beginning $R_{a b}$ and the end $R_{a e}$ in the axis coordinate frame can be calculated by equation (9).

$$
R_{a b}=R_{a} R_{b} \quad R_{a e}=R_{a} R_{e}
$$

\footnotetext{
${ }^{8}$ We do not intend to divide the motion into translation and rotation. Such a motion can be calculated to solve the redundancy of the equation (8).

${ }^{9}$ When $n_{e}=0$, the rotation obtained from observation is the essential one.
} 
Table 1: Transformation coordinates (the sign of the vector $\mathbf{r}_{\mathbf{1}}$ is selected to create a right-hand coordinate system, and the vector $\mathbf{a}$ is $\mathbf{r}_{\mathbf{2}} \times \mathbf{r}_{\mathbf{1}}$ )

\begin{tabular}{|c|c|c|c|c|}
\hline$n_{e}$ & $n_{s}$ & x-axis & y-axis & z-axis \\
\hline \hline 1 & 2 & $\mathbf{r}_{\mathbf{1}}$ & $\mathbf{a}$ & $\bigcirc \mathbf{r}_{\mathbf{2}}$ \\
\hline 1 & 3 & $\pm \mathbf{r}_{\mathbf{1}}$ & $\bigcirc \mathbf{r}_{\mathbf{2}}$ & $\bigcirc \mathbf{r}_{\mathbf{3}}$ \\
\hline 2 & 3 & $\pm \mathbf{r}_{\mathbf{1}}$ & $\mathbf{r}_{\mathbf{2}}$ & $\bigcirc \mathbf{r}_{\mathbf{3}}$ \\
\hline
\end{tabular}

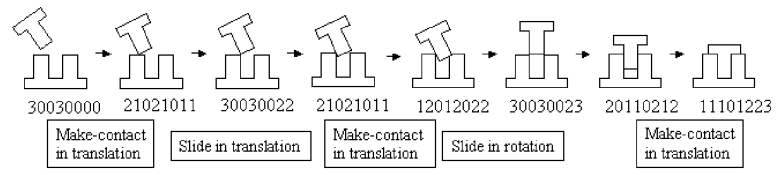

Figure 10: Result of assembly-task recognition

Next, the displacement matrix $R_{b e}$ between $R_{a b}$ and $R_{a e}$ can be calculated by equation (10).

$$
R_{b e}=R_{a b} R_{a e}^{-}
$$

Then, the matrix $R_{b e}$ is converted to RPYrepresentation $(r, p, y)$. The essential rotation is one for which the amount of rotation around the axes as shown $\bigcirc$ in Table 1 becomes zero.

\section{Experimental result}

We implemented the proposed method and verified its validity.

First, we performed peg-insertion in front of the robot vision system. Next, we converted the performance to sub-skill representation as shown in Figure $10[8]$.

Then, we calculated the optimal trajectories. The result is shown in Figure 11.

\section{Conclusion}

We proposed a method to calculate optimal trajectories from sub-skill representations[8]. Calculating optimal trajectories is very difficult, because it is equivalent to solving a system of nonlinear equalities. However, we can overcome this difficulty by calculating the rotation axis direction at first.

\section{Acknowledgments}

This work is supported in part by the Japan Science and Technology Corporation (JST) under the Ikeuchi CREST project, and in part by the Grant-in-Aid for Scientific Research on Priority Areas (C) 13224025 of the Ministry of Education, Culture, Sports, Science and Technology.

\section{References}

[1] K. Ikeuchi and T. Suehiro : "Toward an assembly plan from observation part i: Task recognition with polyhedral objects," IEEE Trans. on Robotics and Automation, Vol. 10, No. 3, 1994.

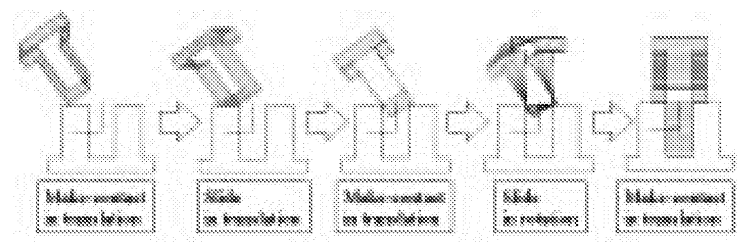

Figure 11: Optimal trajectory

[2] M. Tsuda, T. Takahashi, and H. Ogata : "Generation of an assembly-task model analyzing human demonstration," Journal of the Robotics Society of Japan, Vol. 18, No. 4, pp. $535-544,2000$.

[3] H. Onda, T. Ogasawara, H. Hirukawa, K. Kitagaki, A. Nakamura, and H. Tsukune : "A telerobotics system using planning functions based on manipulation skills and teaching-by-demonstration technique in vr," Journal of the Robotics Society of Japan, Vol. 18, No. 7, pp. 979 $994,2000$.

[4] Y. Kuniyoshi, M. Inaba, and H. Inoue : "Learning by watching: Extracting reusable task knowledge from visual observation of human performance," IEEE Trans. on Robotics and Automation, Vol. 10, No. 6, Dec. 1994.

[5] C. G. Atkeson and S. Schaal : "Robot learning from demonstration," Inter. Conf. on Machine Learning, Vol. 2, No. 12, pp. 12 - 20, 1997.

[6] H. Miyamoto, S. Schaal, F. Gandolfo, H. Gomi, Y. Koike, R. Osu, E. Nakano, Y. Wada, and M. Kawato : "A kendama learning robot based on bi-directional theory," Neural Networks, Vol. 9, No. 8, pp. 1281 - 1302, 1996.

[7] S. Schaal : "Is imitation learning the route to humanoid robots?," Trends in Cognitive Sciences, Vol. 3, pp. 233 242,1999 .

[8] J. Takamatsu, H. Tominaga, K. Ogawara, H. Kimura, and K. Ikeuchi : "Extracting manipulation skills from observation," IEEE Inter. Conf. on Intelligent Robots and Systems, Vol. 1, pp. $584-589,2000$.

[9] T. Lozano-Perez, M. T. Mason, and R. H. Taylor : "Automatic synthesis of fine-motion strategies for robtics," IEEE Inter. Journal of Robotics Research, Vol. 3, No. 1, pp. 3 24, 1984 .

[10] L. E. Kavraki, P. Svestka, J. Latombe, and M. H. Overmars : "Probabilistic roadmaps for path planning in high-dimensional configuration spaces," IEEE Trans. on Robotics and Automation, Vol. 12, No. 4, pp. 566 - 580, Aug. 1996.

[11] H. Hirukawa : "On motion planning of polyhedra in contact," WAFR, 1996.

[12] X. Ji and J. Xiao : "Planning motion compliant to complex contact states," IEEE Inter. Conf. on Robotics and Automation, pp. 1512 - 1517, 2001.

[13] M. S. Ohwovoriole and B. Roth : "An extension of screw theory," Journal of Mechanical Design, Vol. 103, pp. 725 -735 , Oct. 1981.

[14] E. Rimon and J. Burdick : "Mobility of bodies in contact - i: A new 2nd order mobility index for multiple-finger grasps," IEEE Inter. Conf. on Robotics and Automation, pp. 2329 - 2355, 1994.

[15] T. Suehiro and K. Ikeuchi : "Towards an assembly plan from observation: Part ii: Correction of motion parameters based on fact contact constraints," IEEE Inter. Conf. on Intelligent Robots and Systems, pp. 2096 - 2102, Jul. 1992. 